\title{
LA ESTÉTICA DEL PADRE JESUITA PEDRO JOSÉ MÁROUEZ EN SU DISCURSO TITULADO SOBRE LO BELLO EN GENERAL*
}

Juan Granados Valdéz $z^{* *}$

\section{Resumen}

La obra del padre Pedro José Márquez ha sido poco estudiada. Las historias y los estudios de pensamiento novohispano más asequibles no lo incluyen, aunque no ha dejado de haber, empero, recuerdo y crítica del "docto mexicano". Se entiende por Estética, en general y propiamente dicha, la rama de la filosofía que se aboca al estudio de la (propia) sensibilidad que, orientada por el intelecto, es el aparato con el que se capta la belleza. Así pues, la Estética es, en primer lugar, filosofía de la sensibilidad y filosofía de la belleza. Pero la Estética conecta con las artes. Así pues, la Estética, en segundo lugar, es filosofía del arte. Este es el camino que siguió el padre Pedro José Márquez. El propósito de este trabajo es demostrar que la Estética del padre jesuita, especialmente en su discurso titulado Sobre lo bello en general, es una filosofía de la sensibilidad, de la belleza y del arte. Se aprovecharán los

* Este artículo filosófico es producto de la lectura e investigaciones que ha hecho el autor sobre el discurso del padre Jesuita Pedro José Márquez titulado Sobre lo bello en general. Citar como: Granados, J. (2018). La estética del padre jesuita Pedro José Márquez en su discurso titulado sobre lo bello en genera. Cuadernos de Filosofia Latinoamericana, 39(118), 95-114. DOI: https://doi.org/10.15332/10.15332/ s0120-8462.2018.0118.06

* Doctor en Artes por la Universidad de Guanajuato, México, actualmente trabaja en la Universidad Autónoma de Querétaro, México. Correo electrónico: juan.granados@uaq.mx 
recursos teóricos y prácticos que aporta la hermenéutica analógica. Este trabajo estará dividido en dos partes. En la primera se presentan los contextos de Sobre lo bello en general; en la segunda se trata la Estética del padre jesuita.

Palabras clave: estética, sensibilidad, belleza, arte, Pedro José Márquez.

\section{Abstract}

The work of Father Pedro José Márquez has been little studied. The most accessible histories and studies of Novohispano thought do not include it, although there has nonetheless been a memory and criticism of the "mexican doctor". Aesthetics is understood, in general and properly speaking, the branch of philosophy that focuses on the study of (own) sensibility, which, guided by the intellect, is the device with which beauty is captured. So the Aesthetics is, in the first place, philosophy of the sensibility and philosophy of beauty. But the Aesthetic connects with the arts. So, Aesthetics, secondly, is philosophy of art. This is the path that Father Pedro José Márquez followed. The purpose of this paper is to demonstrate that the Jesuit Father's Aesthetics, especially in his speech entitled On the beautiful in general, is a philosophy of sensibility, beauty and art. The theoretical and practical resources provided by Analogical Hermeneutics will be taken advantage of. This work will be divided into two parts. In the first, the contexts of On the beautiful in general are presented; and, in the second, the Aesthetics of the Jesuit father will be treated.

Keywords: Aesthetics, sensibility, beauty, art, Pedro José Márquez.

\section{Resumo}

A obra do padre Pedro José Márquez tem sido pouco estudada. As histórias e os estudos do pensamento novohispano mais acessíveis não o incluem, ainda que, não tem deixado de haver, porém, uma lembrança e critica do "douto mexicano". Entende-se por Estética, no geral e propriamente dita, a área da filosofia que se ocupa do estudo da (própria) sensibilidade que, orientada pelo intelecto, é o aparato pelo qual se capta a beleza. Assim, a estética é, em primeiro lugar, filosofia 
da sensibilidade e filosofia da beleza. Mas a Estética conecta com as artes. Assim, a Estética, em segundo lugar, é a filosofia da arte. Este é o caminho seguido pelo padre Pedro José Márquez. O propósito deste trabalho é demonstrar que a Estética do padre jesuíta, especialmente no seu discurso titulado Sobre o belo em geral, é uma filosofia da sensibilidade, da beleza e da arte. Se aproveitarão os recursos teóricos e práticos que aporta a hermenêutica analógica. Este trabalho estará divido em duas partes. Na primeira se apresentam os contextos de Sobre o belo em geral; na segunda trata-se a Estética do padre jesuíta.

Palavras-chave: estética, sensibilidade, beleza, arte, Pedro José Márquez.

\section{Introducción}

La obra del padre Pedro José Márquez (PJM) ha sido poco estudiada. Su figura sigue siendo poco conocida. Las historias y los estudios de pensamiento novohispano más asequibles no lo incluyen (Beuchot, 1996; Navarro, 1998; Larroyo, 1989; Ibargüengoitia, 1989; Ibargüengoitia, 1982). Aunque no ha dejado de haber, empero, recuerdo y crítica del "docto mexicano" (Fernández, 1972), incluso en fechas próximas.

El más reciente intento por rescatar la figura y época del padre PJM fue el de Óscar Humberto Flores, investigador del Instituto de Investigaciones Estéticas de la UNAM, en colaboración con el Instituto y la Real Academia de Bellas Artes de San Fernando, España, que fue sede y quien organizó del 27 al 30 de octubre de 2009 el coloquio internacional "El clasicismo en la época de PJM (1741-1820). Arqueología, filología, historia y teoría arquitectónica". Participaron especialistas de distintos países, y se publicó un volumen con las intervenciones y otros estudios complementarios (Astorgano, 2015a). Esto motivó aún más el estudio del padre jesuita que, en palabras del propio Óscar Flores, resulta más conocido en Europa, quizás porque la mayor parte de su obra está en italiano, que en México (Flores, 2010).

Lo anterior, la precaria situación por la que pasa la Estética, despedida y desahuciada por algunos, además de este extraño y reciente aniversario de hace 250 años, el de la expulsión de los jesuitas de los territorios españoles y su disolución han movido a preparar este trabajo sobre la Estética del padre jesuita PJM.

* Este artigo filosófico é produto da leitura e pesquisas que tem feito o autor sobre o discurso do padre jesuita Pedro José Márquez titulado Sobre o belo em geral. 
Se entiende por Estética, en general y propiamente dicha, la rama de la filosofía que se aboca al estudio de la (propia) sensibilidad (Arvizu, 2005; Beuchot, 2012), base de la experiencia y el juicio estéticos, y que, orientada por el intelecto, es el aparato con el que se capta la belleza. Así pues, la Estética es, en primer lugar, filosofía de la sensibilidad y filosofía de la belleza. Pero la Estética conecta con las artes, ya que estas son objeto de la belleza y motivan especialmente a la sensibilidad. La Estética, en segundo lugar, es filosofía del arte. En esta parte se distinguen la creación artística y sus condiciones y la recepción de las artes y sus condiciones. El estudio del gusto resulta del tratamiento de lo anterior (Beuchot, 2012; Blanco, 2007). A esta definición y sus detalles quisiera sumar que hay dos caminos de acceso a la Estética, a saber: uno ascendente y otro descendente. El primero va de los hechos a las ideas, de lo particular a lo general, de la práctica a la teoría. Es el camino de la inducción. El segundo sería el de la deducción, el que va de lo general a lo particular, de la teoría a la práctica. La Estética se mueve entre ambos caminos, aunque, por ser primero, saber de la sensibilidad sea más fácil a través del camino ascendente, por paradójico que suene (Blanco, 2007, p. 19). Y como veremos este es el camino que siguió el padre PJM.

En el marco de lo dicho, pues, sobre la Estética el propósito de este trabajo es demostrar que la Estética del padre jesuita, especialmente en su discurso titulado Sobre lo bello en general, es una filosofía de la sensibilidad, de la belleza y del arte. Para conseguir este objetivo se aprovecharán los recursos teóricos y prácticos que aporta la hermenéutica analógica (Beuchot, 2009) que entre otras cosas propone que interpretar es comprender (y explicar) poniendo un texto en sus contextos.

Este trabajo estará dividido en dos partes. En la primera se presentarán los contextos de Sobre lo bello en general, a saber, los contextos culturales (filosóficos y artísticos) y biográficos; en la segunda se tratará la Estética del padre jesuita. Se cerrará el escrito con algunas ideas que serán consideraciones sintéticas de la exposición que la antecede.

\section{Contextos}

\subsection{Contextos culturales}

Me detendré en la presencia jesuita en México, en su labor filosófica y educativa, y en la cultura artística del periodo del padre PJM. Para nuestro propósito, ambos son contextos imprescindibles para comprender la Estética de nuestro autor. Después 
pasaré revista a su vida y enumeraré algunas de sus obras, antes de tratar Sobre lo bello en general.

La Compañía de Jesús (Societas Jesu, S. J.) o los jesuitas, como orden fue fundada en 1534 por San Ignacio de Loyola y otros en Roma. Fue aprobada por S. S. Paulo III en 1540. El 9 de septiembre de 1572 los jesuitas, a través de Veracruz, arribaban a la Nueva España, y el 28 del mismo mes llegaban a Ciudad de México. Alonso de Villaseca les otorgó unos solares detrás de la Catedral Metropolitana. Allí fundaron el Colegio Real y más Antiguo de San Ildefonso, cuyo edificio es cumbre del barroco mexicano. No fue el único colegio fundado por la Orden en México. Están también los Colegios de San Ignacio y San Francisco Javier, en Querétaro.

En el ámbito de la enseñanza de la filosofía, en 1577, llegó a México Antonio Rubio (1548-1615), nacido en Villa de Rueda, España. Fue enviado por sus superiores para enseñar filosofía y teología. Vuelve a Europa en 1600. El proyecto filosófico y educativo de Rubio era un Curso de filosofía que abarcaba lógica, física y metafísica. Aunque era un curso, buena parte de él se dedicó a comentar al estagirita. Otro notable jesuita, del siglo XVI, fue Antonio Arias (1565-1603). Hizo anotaciones a los comentarios del Card. Francisco de Toledo; comentó los libros de física, del cielo y meteorológicos de Aristóteles. Dejó un tratado de filosofía del lenguaje, denominado De lingua originali. En el siglo XVII destacaron los padres jesuitas Andrés de Valencia, que escribió sobre lógica; Alonso Guerrero, que dejó un comentario a los libros de la física del estagirita; Diego Marín de Alcázar y Agustín Sierra, quienes dejaron cursos de filosofía. El siglo XVIII se divide entre la Escolástica y la incorporación y promoción del pensamiento moderno. De la primera mitad de este siglo, entre los jesuitas escolásticos, fueron notables José Maldonado, José Ignacio Sánchez y Francisco Javier Alejo de Orrio. La segunda mitad del siglo XVIII se caracteriza por un afán de asimilar las ideas filosóficas y científicas modernas. Esto trajo consigo una renovación contra la tradición que se dio en la Universidad y en los colegios religiosos, especialmente en los de los jesuitas. De hecho, el siglo XVIII fue el más intenso para los Colegios de la Compañía de Jesús, hasta su expulsión en 1767 (Beuchot, 1990). No es exagerado decir que los jesuitas fueron los educadores de la juventud mexicana e influyeron mucho en la filosofía, tanto en la tradicional como en la introducción de las ideas modernas (Beuchot, 1996, p. 219). 
En el marco de este contexto filosófico y educativo se formó el padre PJM, al menos hasta antes de partir a Europa, a partir de la expulsión de la Compañía de Jesús (fundada en la Pragmática de Carlos III), con muy poca edad y sin haber profesado.

Habiendo repasado sucintamente el marco jesuita educativo y filosófico, es importante destacar algo acerca del contexto artístico de la época del padre PJM. El interés por las artes entre los jesuitas no será menor, de entre los más de mil jesuitas expulsados, una docena eran artistas y escribieron sobre música, pintura y arquitectura. Destacan como musicólogos Antonio Eximemo y Esteban de Arteaga. Pero dos estudiosos del arte grecolatino en pleno neoclasicismo, apenas y son recordados, a saber, PJM (1741-1820), experto en arquitectura, y Vicente Requeno (1743-1811), experto en música, pintura, pantomima y telegrafía óptica (Astorgano, 2015b, p. 191).

Con la Ilustración del siglo XVIII se buscaron de nuevo para las artes los principios del (arte) clásico, surgiendo el neoclásico como reinterpretación del arte grecolatino. Reconocer el mundo antiguo también motivó la escritura de teorías. La arquitectura fue una de las artes que más atención mereció, y por la especialidad del PJM será sobre ella que ahondaremos más. Vitruvio, que no había sido abandonado, se revisó más asiduamente. El padre Joseph Ortiz y Sanz, en 1787, indicó que para entender a Vitruvio era necesario saber latín, no ignorar el griego, habituarse a la frase vitruviana, conocer el arte arquitectónico de manera teórica y práctica y tener conocimiento de muchas otras ciencias como la física, la historia, la música, la geometría, el dibujo, etc. Esta fue una de las preocupaciones de la Ilustración, integrar las ciencias y las artes.

Uno de los tratadistas más importantes en este sentido fue Claude-Nicolas Ledoux, quien en su obra L'architecture considérée sous le rapport de l'art moeurs et de la législation, publicada en 1804, creó un estilo "basado en la expresión arquitectónica de los nuevos ideales sociales y los descubrimientos científicos". (Fernández, 2011, p. 546)

Además, el interés por lo antiguo no se limitó al mundo clásico, sino al mundo entero. Johann Bernhard Fisher von Erlack, en su Plan de arquitectura histórica y civil, de 1721, realiza estudios de culturas comparadas. En medio de la efervescencia de conocimientos se dio la necesidad de llevar al arte al primer estilo internacional, que consistió en una uniformidad universal apoyada en el arte clásico. Las academias de arte fueron clave para esto. El objetivo de las academias neoclásicas era combatir los desórdenes artísticos del barroco (Fernández, 2011). 
La perspectiva de integrar artes y ciencias como la de comparar culturas la compartió el padre jesuita PJM, en su esfuerzo por revalorar la arquitectura prehispánica y virreinal. Es el momento de hablar acerca de él.

\subsection{Biografía, obras y pensamiento}

Antes de entrar en materia estética se referirá entonces para cumplir con el propósito de este escrito, algunos datos de la biografía de este autor (Flores, 2010).

El padre jesuita PJM nace en el año de 1741 en Rincón de León, Guanajuato, México. Como miembro de la Compañía de Jesús fue expulsado en 1767 junto con muchos otros. Se estableció en Roma durante su exilio. Al igual que Francisco Javier Clavijero, José Lino Fábrega y Andrés Cavo, se dedicó a dar a conocer la historia antigua de México para reivindicar su patria de los ataques emprendidos durante la llamada Disputa del Nuevo Mundo. Por sus obras alcanzó un amplio reconocimiento en Europa, entre anticuarios y eruditos, y entre artistas e intelectuales. Estos conformaban el círculo ilustrado del embajador español en Roma, José Nicolás de Azara. PJM fue nombrado socio honorario de la Real Academia de Bellas Artes de San Fernando y de la Real Academia de Nobles y Bellas Artes de San Luis de Zaragoza. Mantuvo vínculos con la Real Academia de Bellas Artes de San Carlos de Valencia. En Italia formó parte de las academias de Bellas Artes de Bolonia, Florencia y de la arqueológica de Roma. Con el restablecimiento, por orden de Fernando VII, de la Compañía de Jesús en 1816, PJM volvió a México. Fue nombrado rector de los Colegios de San Pedro y San Pablo de la ciudad de México. José Bernardo Couto, alumno suyo, fue también su primer biógrafo (Diccionario Universal de Historia y Geografía, 1854). PJM falleció en la Ciudad México en 1820.

La producción reflexiva del padre Márquez es vasta. A partir de sus estudios filológicos y su participación en excavaciones para rescatar vestigios romanos, publicó ocho obras en italiano y una en español, relacionadas con la astronomía, la arqueología, la historia y las artes de la antigüedad clásica y del México Prehispánico. A continuación se enlistan dichas obras:

- Tavole nelle qualle si mostra il punto del mezzo giorno e della mezza notte, del nascere e tramontare del sole, secondo il meridiano di Roma per regolare orologi all'italiana e dalla francese (Tablas en las que se muestra el mediodía y 
la medianoche, el amanecer y la puesta del sol, de acuerdo con el meridiano de Roma para ajustar relojes italianos y franceses), de 1790.

- Delle case di citá degli autichi Romani, secondo la dottrina di Vitruvio (De las casas de este antiguo romano, según la doctrina de Vitruvio), de 1795.

- Delle ville di Plinio il giovane, con un appendice su gli atrii della S. Scritura, e gli scamilli impari di Vitruvio (Las villas de Plinio, el joven, con un apéndice en los atrios de S. Scritura, y los cabrones aprendidos por Vitruvio), de 1796.

- Dell'ordine dorico (Del orden dórico), de 1803.

- Sobre lo bello en general, de 1801.

- Saggio dell'Astronomia, Cronologia, e Mitologia degli antichi Messicani (Saber de astronomía, historia y mitología de los antiguos mexicanos), de 1804.

- Due Antichi Monumenti di architettura Messicana (Dos monumentos antiguos de la arquitectura mexicana), de 1804.

- Esercitazioni architettoniche sopra gli spettacoli degli antichi, con appendice sul bello in generale (Ejercicios arquitectónicos sobre los espectáculos de los antiguos, con apéndice sobre lo bello en general), de 1808 .

- Ilustrazioni della villa di Mecenate in Tivoli (Ilustraciones de la villa de Mecenate en Tivoli), de 1812.

Aún siguen sin traducirse algunos de estos textos. Incluso hay obras todavía inéditas. De entre ellas destaca ese interés compartido por el teórico romano de la arquitectura:

- Apuntamientos por orden alfabético pertenecientes a la arquitectura de Marco Vitruvio Pollión, que trabajó desde 1784 hasta 1812.

Mencionadas las obras del PJM, se adelantará algún comentario sobre su pensamiento en el marco del humanismo, tal como lo ubican quienes lo han estudiado. Hay dos temas frecuentes o intereses esenciales en las obras del padre PJM y que se notan tan solo de los títulos de las obras que recién se enumeraron. Estos son, en primer lugar, la exposición de la cultura mexicana en Europa, que como ya se mencionaba es un interés que comparte con otros correligionarios al participar en la Disputa del 
Nuevo Mundo, y el humanismo, tema también frecuente en otras plumas jesuitas. La exposición de la cultura mexicana en PJM se da como una necesidad. Plantea, con ello, el tema de la identidad novohispana, base de la mexicana. No por nada firmaba sus obras en italiano como "Pietro Márquez, Messicano".

Aunque sus padres fueran españoles, arraigo y sentimiento de pertenencia los desarrolla por su patria o nación. Defendió a los mexicanos de su época, que jugaban en la comedia del mundo, el papel de plebe, defendiendo a los mexicanos antiguos, que fueron amos, con una cultura y una educación de renombre.

Márquez reconoce a los antiguos mexicanos como los antepasados de los nacidos en México. Más adelante, vuelve a referirse a la nación mexicana al nombrar las luces que tenía; hace referencia a la nación mexicana, situada en el gran país del Anáhuac llamado actualmente Nueva España. (Rovira, 2014, p. 128)

PJM protege su cultura y critíca todos los intentos europeos de minusvalorar la cultura indígena, sea por el factor que sea, por eso promueve, entre otras cosas, que los europeos conozcan el arte prehispánico. Para PJM todos somos humanos, seamos indígenas, criollos o europeos.

Su "humanismo" (el de Pedro Javier Alegre y PJM) es el respeto absoluto "al otro" y "el reconocimiento de sus derechos como hombre"; este humanismo no está basado en la caridad ni en la fantasía, sino en la realidad social concreta en que vivían. (Rovira, 2014, p. 118)

Defiende, porque descubre la naturaleza humana en todos, al ser humano concreto, especialmente al desprotegido, sin que importe su color de piel o la lengua que hable.

Sobre la arquitectura, de la que es o se le reconoce como especialista, el padre PJM es un teórico como cualesquiera de los teóricos europeos de la época. Es un ilustrado, como ya se decía, que busca integrar artes y ciencias y abrir la reflexión a otros ámbitos del mundo. Sus ideas sobre esta arte conectan con su humanismo y su interés de reivindicar a los mexicanos. A continuación presento algunas de sus ideas en función de que uno de los objetivos de sus estudios arquitectónicos fue ponderar la arquitectura mexicana prehispánica y virreinal al mismo nivel que la europea. Martha 
Fernández (2011), con su artículo titulado "Literatura artística en el siglo XVIII", me orienta en la comprensión de este objetivo. PJM en su Dell'oridinde dorico dice:

\begin{abstract}
¿Quién negará, por ejemplo, que los antiquísimos etruscos sin haber tenido noticia de Doro, alzaron muchos edificios con columnas, las cuales han creído algunos eran de orden toscano? En cuanto a los egipcios y otras naciones orientales, ninguno puede negar que las usaban antes de los griegos... Los mexicanos también, gente desconocida por tantos siglos, y que no oyó jamás el nombre de Doro, usaban columnas en sus edificios y no de madera, sino también de piedra y finalmente sin quitarle el mérito a los otros pueblos que como los doros y sin aprender de ellos se fabricaron sus edificios... siendo conveniente fijar algún nombre para entendernos... llamaremos dórica la manera de edificar, que en Grecia... [y] por razones de su parecido llamaremos también dórica a la manera de edificar de todos aquellos pueblos. (Márquez,
\end{abstract} 1803 , p. 138)

Como sostiene Juana Gutiérrez (1988), lo que hace PJM no es regatear la autoría del dórico a los griegos, sino la autoría de la arquitectura clásica y así hacer de lo clásico una invención humana (asumida después por los griegos y codificada por Vitruvio). De esta manera la arquitectura mexicana se incorporaba por derecho al arte clásico. Lo mismo que Fisher vin Erlack compara las arquitecturas occidentales y del resto del mundo cuando relaciona el lacónico con el temazcalli:

Y quién sabe si los primeros padres de los mexicanos trajeron del mundo antiguo el uso de estos baños vaporosos, como sus actuales descendientes los conservan todavía como era su primitivo origen; mientras los griegos y los romanos habiéndolos tomado de la misma fuente, primero los cambiaron y después los abandonaron. Que si es así ya que de estos no podemos en el presente aprender cómo era el lacónico, qué maravilla que aquellos del otro mundo nos lo puedan enseñar. (Gutiérrez, 1988)

Además, desde las tres características de la arquitectura clásica, solidez, comodidad y belleza, PJM analiza la producción arquitectónica de la Nueva España. En una Historia de la arquitectura, aún inédita: 
Por lo que toca a la belleza, los órdenes griegos se ponen en ejecución conforme a la práctica de nuestros tiempos, quiere decir a veces con buen gusto, a veces no: las fachadas de los edificios se decoran variamente así en lo externo como en lo interno: el uso de los mármoles es poco, pero habiendo en muchas partes abundancia de cantería ya de un color, ya de otro, se vale de ella oportunamente, como en Roma de los travertinos: con esta fabrican muy lindas paredes de sillería; y también con ladrillos, con sillarejos y otros materiales saben adornar las fachadas respectivas con un gusto natural y sencillo. El tezontle de México, que es una piedra esponjosa, consistente y de buen color conduce mucho para la belleza del externo de los edificios, y de la simetría de las casas y sus fachadas, con igualdad de las calles, y variedad de las fábricas constituye un bello conjunto en las más de las poblaciones ${ }^{1}$. (Márquez, 1988)

Con lo anteriormente señalado este trabajo ya puede encaminarse al tema que que se ha propuesto tratar, el de la Estética de PJM en su discurso titulado Sobre lo bello en general.

\section{La Estética de PJM en Sobre lo bello en general}

El discurso Sobre lo bello en general fue escrito por PJM en 1801. Suma 31 páginas y se divide en 15 parágrafos o apartados que se numeran, pero no se titulan. Lo antecede una misiva dirigida simplemente a un "Amigo". En ella cuenta que después de exponerle los puntos de la arquitectura teórica, a partir de una temática bíblica, los atrios que en la escritura "se mientan", el amigo se enteró que trabajaba en algo sobre lo bello en la arquitectura y lo instó a escribir una obra. Las vicisitudes de su vida lo habían impedido. Y ya que tuvo tiempo la obra esperada no se dio, ya que en el proceso del intento por tratar lo bello en la arquitectura, indicó "que por las mismas razones que son bellas las obras de esta arte, lo es también qualquiera otra cosa que se pueda decir bella" (Márquez, 1801, p. 3). De esta manera resultó este discurso sobre lo bello general, que a la larga sirviera para "inferir los particulares principios de donde venga el que sea bella la arquitectura” (Márquez, 1801, p. 4). Y

1 Juana Gutiérrez Haces, en Historia, leyendas y mitos de México: Su expresión en el arte. (XI Coloquio Internacional), México, Universidad Nacional Autónoma de México, 1988. Esta cita fue tomada de la Historia de la arquitectura, manuscrito del padre Márquez, descubierto por Juana Gutiérrez Haces en Italia hace algunos años. El manuscrito permanece inédito debido al desafortunado fallecimiento de la maestra Gutiérrez Haces. 
ya con esto dicho se está entrando en materia. Se decía que la Estética es filosofía de la sensibilidad, filosofía de la belleza y filosofía del arte. PJM anuncia en este pórtico que el descubrimiento es que la belleza lo es de todo lo que es bello y no solo de un arte $\mathrm{u}$ otra, porque por las mismas razones todas lo son. Esas razones se descubrieron en el "intento" que menciona. Ese intento parte de la experiencia y lo que faculta tal experiencia es la sensibilidad, en la que se reúnen percepción e inteligencia. Puede decirse que el camino es inductivo. Y ya que se tiene una idea de belleza se pueden inferir los particulares de la belleza de cualquier arte. Aquí el camino se vuelve deductivo. Y estos son los caminos de la Estética que se mencionaban, el ascendente y el descendente. Y ambos se encuentran en la obra de PJM. Anunciadas, en estas palabras introductorias al discurso, las tres perspectivas de la Estética según se trate de la sensibilidad, la belleza o las artes, se tratarán en la obra estudiada cada una de ellas.

\subsection{Filosofía de la sensibilidad}

PJM del parágrafo VIII al XIV expone su filosofía de la sensibilidad (Márquez, 1801, pp. 16-28), que de entrada debe quedar claro, no debe confundirse con solo la percepción sensible. En la sensibilidad se integran también emoción, imaginación e inteligencia (Beuchot, 2012, p. 14). A continuación se la presentará.

El espíritu goza con el placer que le producen las cosas bellas, especialmente con las artes que fueron hechas para tales efectos. Estas comunican sus ideas por medio de los oídos y los ojos. Son estos, pues, la vía de comunicación del espíritu con dichos objetos. En otras palabras, el espíritu consigue complacerse en los objetos bellos a partir de las impresiones sensibles que obtiene de estos dos sentidos. El acto de complacerse del espíritu con las artes se subdivide en tres o tiene tres circunstancias, según expone PJM.

El primer acto o primera circunstancia del acto es, pues, ver y oír. Pero aquí PJM se percata que, si bien es el primer acto para la complacencia del espíritu, hay quien oyendo o viendo composiciones poéticas no reflexionan, ora por distracción, ora por falta de inteligencia. Entonces ver y oír materialmente no basta. El segundo acto (o la segunda circunstancia del acto) es que ver y oír deben hacerse con atención racional. Pero todavía no es suficiente. Hay quienes estando presentes en la ejecución de una sinfonía y prestando toda su atención, son incapaces de deleitarse con ella, porque no tienen oído para eso, es decir, no saben discernir lo que oyen. El tercer acto o tercera circunstancia es que el acto de ver y oír sea con discernimiento. Cuando la vista y el oído se acompañan de atención y discernimiento, este lleva consigo los actos del 
entendimiento y la voluntad. Así el espíritu, a partir de un buen oído y una buena vista (la sensibilidad orientada por la inteligencia), consigue ese placer que solo los objetos bellos pueden proporcionarle.

El buen oído y la buena vista son lo que se llama en las bellas artes, dice PJM, buen gusto. Sobre este hay algunas cosas que decir. En primer lugar, aquellos pueden ser naturales o adquiridos. Si son naturales se perfeccionan con los hábitos y las reglas. Si son adquiridos tendrán que vencerse las dificultades por medio de los hábitos y las reglas. Los primeros se engendran de los actos de la misma especie continuados y convenientes. Las reglas se orientan según el propósito. Este es el fin de los hábitos que se consiguen siguiendo las reglas. Dependerá de si se busca enseñar o aprender a ver con buenos ojos una pintura o con buenos oídos escuchar una canción. El gusto se perfeccionará con el uso y conocimiento de hábitos y reglas. En segundo lugar, el buen gusto será más o menos extenso según la cantidad de objetos que se puedan discernir y será más o menos perfecto según la perspicacia con que se vean u oigan los mismos objetos. Esto lo dice PJM porque, por ejemplo, es claro que el buen oído no lo es para todo lo que puede ser percibido por este, es decir, quien tiene o ha adquirido buen oído para la música no lo tiene para la elocuencia. En tercer lugar, el buen gusto, que es metáfora del sentido del gusto, sí, pero como este, es capaz, tiene la aptitud porque sabe, de sentir inmediatamente o al instante que está frente a una dulzura o una belleza. Lo que requiere el buen gusto para serlo es la aptitud que se gana con el conocimiento que se obtiene por la experiencia (hábito).

A las anteriores consideraciones PJM dice que el buen gusto se requiere para conocer la belleza de las artes, pero también para percibir la belleza en la naturaleza. Los principios que fundarían esta percepción son distintos y producen en nosotros cierta persuasión que puede ser doblegante. Esta persuasión puede ser interna o externa. Es interna cuando los principios son innatos o influyen sin que precedan discursos o raciocinios, como cuando se percibe lo hermoso de algún evento natural sin más. Es externa la persuasión cuando los principios son precedidos por discursos o raciocinios. Estos principios son comunes a todas las naciones. Un ejemplo de ello son las ideas respecto a la perfección física de los rostros. También influyen las ideas nacionales. PJM dice:

Pues como influyen las ideas nacionales en el gusto de cada pueblo, así influyen las costumbres comunes, las tradiciones antiguas, las máximas generales, y todos quantos principios se hallen ser adoptados por la muchedumbre, en los 
quales (por condicion necesaria) no tengan que hacer las pasiones; porque aquellos, como fundados en razon, y no estas, que por parciales siempre deben excluirse, son los que causan la persuacion externa que hemos dicho, de que los obgetos sensibles de la naturaleza son bellos. (Márquez, 1801, p. 21)

Como puede notarse, en la filosofía de la sensibilidad de PJM sí se integran la percepción, la emoción, la imaginación y la inteligencia. Aunque se reduzca a dos sentidos y se promueva el buen gusto, estas ideas no adolecen de vigencia. La aceptación de que las ideas nacionales influyen en los gustos de los pueblos parece adelantarse a su época en muchos sentidos, pues precede o los estudios del marxismo sobre la ideología o los estudios hermenéuticos sobre los prejuicios. Pero para ampliar de más esto, se da paso ya a la filosofía de la belleza.

\subsection{Filosofía de la belleza}

La definición que aporta el padre jesuita PJM de la belleza o de lo bello la plantea a modo de conclusión, pero en forma de pregunta:

¿Y no nos será lícito concluir de ahí, que como no puede haber bello, que no sea perfecto en verdad y bondad; tampoco pueda serlo lo que al espíritu no sea agradable? ¿Y que por eso lo que al concepto de la belleza debe concurrir es, perfeccion de parte del obgeto, y agrado movido por la perfeccion de parte del espíritu? (Márquez, 1801, p. 29)

En esta definición de belleza puede verse cómo confluyen la ontología y la epistemología en las que toda estética se funda, ya que, por un lado, lo bello se reconoce en los objetos por su perfección y, por otro lado, por el agrado que producen en el espíritu. Esto último se desprende de la comprensión y de la sensibilidad a partir de la participación de los sentidos en la aprehensión de la belleza por el espíritu.

A la perfección también puede llamársele acabamiento o completitud, decían los escolásticos. Estos mismos, especialmente Tomás de Aquino, sumaban a la perfección las notas de proporción y resplandor. La perfección era una característica necesaria, pero no suficiente; la proporción, necesaria y suficiente; y el resplandor, suficiente pero no necesaria (Beuchot, 2012, p. 41). Todas estas notas remiten a la belleza en sí, especialmente la de la perfección. Por eso que se diga que en esta filosofía de lo bello 
confluye la ontología. Y también confluye porque PJM distingue especies de belleza según orden y jerarquía. Así lo expresa:

Y así Dios, la primera y esencial belleza, por ser fuente y orígen de toda verdad y bondad; de ahí las virtudes, que mas que otros obgetos participan de esas prerogativas de la divinidad; luego las verdades subalternas, en quienes hay tanto de divino; y despues por su órden todos los otros obgetos, que segun su género participan ya mas ya, mcnos de dichas nobles qualidades. (Márquez, 1801, p. 29)

Y puesto que la perfección del objeto mueve al agrado por parte del espíritu, es que se dice que confluye en esta Estética la epistemología. Se sabe de lo bello de la cosa porque es perfecta, pero se sabe que es perfecta porque se constata, en primerísimo lugar, por medio de los sentidos o la percepción o la sensibilidad. Así lo dice PJM respecto a la belleza que se encuentra en la naturaleza, como la que se encuentra en las artes:

Las bellas formas, que la naturaleza nos presenta, son las que ella produce segun los modelos que para la perfeccion de cada género tiene destinados; los que las constituyen mas ó menos hermosas, á proporcion de la liberalidad, con que se íos franquea. Un prado en dias de primavera, enriquecido de yerbas y flores, con aquella graciosa variedad que sola naturaleza sabe repartir; es un obgeto de tan perfecta forma, que visto por nuestros ojos, y por ellos pasando á nuestro espíritu las ideas de su regulada variedad, produce en él la complacencia de su indubitable belleza. Así tambien el canto de un ruiseñor compasado con su natural armonía, es uno de aquellos obgetos naturales, que comunicados al espíritu por medio de los oidos, causan en el mismo la complacencia de su belleza.

En semejante manera las voces artificialmente concertadas, ó por un Orador, $o ́$ por un Poeta, ó por un Músico, y las formas acabadas segun arte, ó por un Pintor, ó por un Escultor, ó por un Arquitecto, son otros tantos obgetos, que tocando aquellos nuestros oidos, y estos nuestra vista, mandan sus ideas á nuestro espíritu, quien conociéndolos conformes á sus respectivas, leyes y reglas, se complace, porque en esto mismo encuentra la belleza. ¿Con quán agradable transporte no se eleva el espíritu al tiempo que resuenan en el oido las bien medidas rimas de una poética composicion, las bien acordadas voces de un 
instrumento músico, ó, los bien ordenados períodos de una eloqüente pieza? ¿No queda encantado el espíritu de un inteligente, á la vista de un quadro pintado de mano maestra, de una estatua por un primario Escultor acabada, de un templo con las mas adaptadas proporciones fabricado? (Márquez, 1801, p. 16)

El espíritu, que aprehende e interpreta las impresiones o datos de esta, es movido al agrado. Es decir, se conoce la perfección de la naturaleza y las artes por el espíritu que se aprovecha de los datos sensibles que obtiene por la sensibilidad. El espíritu se complace en las artes porque encuentra en ellas la perfección. A continuación se entra en su filosofía del arte.

\subsection{Filosofía del arte}

PJM habla de los músicos y de los pintores, por solo mencionar algunos de los artistas referenciados en su discurso. Pero respecto a su filosofía del arte o de las artes, se piensa, esta puede encontrarse en la clasificación que hace de tres géneros de objetos, además de otras ideas. Se trata de los objetos que agradan, sean o no bellos, y cuyo conducto son los sentidos. En función de la definición de bello que sostiene, que un objeto bello es algo que agrada al espíritu, se decidirá si lo objetos revisados cumplen o no con el requisito, y si por tanto, son o no bellos. En esta filosofía del arte confluyen las anteriores.

El primer género, dice, es el de los objetos que producen placer en los sentidos del gusto, el olfato y el tacto. Son los que causan placer puramente sensible y material. Lo sabroso, lo oloroso y lo blando hacen su primera impresión en los sentidos que les corresponden y el placer que causan es animal. Esto se debe a que lo perciben tanto los hombres como las bestias. Por tanto, dice, PJM, dichos placeres no pertenecen al espíritu, para cuyas potencias no fueron hechos. Por tanto, no pueden llamarse bellos, porque la parte racional no se complace en ellos (Márquez, 1801, pp. 7-8).

El segundo género es el de los objetos que corresponden a los sentidos de la vista y el oído. Se distinguen de los anteriores porque los objetos no producen placer directamente en los sentidos, sino que es a través de ellos que el espíritu se complace. El complacerse de la armonía de las voces y la perfección de las formas es propio del espíritu y la racionalidad. Los objetos armónicos y bien formados no se hacen para cuadrar al cuerpo, sino al espíritu. Estos objetos sí son bellos, porque agradan al espíritu (Márquez, 1801, pp. 8-9). Como puede inferirse, se trata de las artes que 
se denominan visuales y sonoras, a saber, la música, la escultura, la pintura, la arquitectura, la poesía y el teatro (y la danza), que son las bellas artes. Esto ya lo había adelantado cuando se exponía la filosofía de la sensibilidad de PJM. Y aunque se nota la herencia occidental de distinguir lo que es arte de lo que no, según se dirija a unos u otros sentidos, porque se privilegian unos y no otros, no es momento de cuestionar eso.

Una cosa más, respecto a las artes. Si estas son perfectas, es decir, ya que son bellas y agradan, entonces son perfectas, PJM se pregunta por qué lo son. Se dijo al principio que la Estética como filosofía del arte se interesa en las condiciones de la creación artística. Entonces, según nuestro autor, las artes obtienen su perfección, que les abona belleza, de cierta capacidad del artista. Así lo dice:

Pues quantos aspiran por acercarse á tan gran Maestro, en sus quadros estudian las mejores leyes de la pintura. Lo que hay es, que tanto Rafael, quanto Ciceron, y quantos ha habido ó pueda haber primarios maestros de bellas artes ó de bellas letras, comprendiendo en su gran mente las leyes todas de sus respectivas facultades, han tenido el talento de elegir las que eran mas del caso, y han poseido el ingenio de saberlas poner en práctica con novedad. Pues de esta sabia eleccion de las leyes, y de la novedad con que sean puestas por obra, y no de otra cosa, decimos que proviene ó resulta el mas sublime bello que pueda imaginarse, sea en lo natural, sea en lo artificial. (Márquez, 1801, p. 22)

Para PJM en el artista coinciden, en palabras de otros teóricos del arte de la época, como Hegel, genio y técnica, talento y saber hacer.

El tercer género de objeto es el de los que causan placer directamente al espíritu porque se perciben de manera inmediata por las potencias de este. Estos son la verdad, la justicia, cualquier virtud, el mismo espíritu, Dios. Por tanto, son innegablemente bellos (Márquez, 1801, pp. 8-9).

\section{Conclusión}

Para finalizar, PJM es heredero del patrimonio educativo y filosófico de los jesuitas. A su regreso de Roma se forma en sus colegios y será rector de uno. Así mismo es heredero del neoclasicismo artístico de los siglos XVIII y XIX. El interés por la arquitectura 
de dicho periodo marca los intereses de este filósofo y esteta. Nace en México, pero su obra escrita, en medio de la polémica y la disputa, en medio del doble descrédito que para él significaba ser mexicano y jesuita, la escribirá en Italia. Defenderá a su pueblo y pregonará un humanismo del otro, como otros correligionarios suyos. $\mathrm{Y}$ aunque la arquitectura, la clásica, la neoclásica, la prehispánica y la virreinal serán de su interés, descubre que la belleza lo es, cuando algo es bello, siempre de la misma forma, sea el arte que sea.

PJM dice que lo bello de un objeto está en su perfección (en verdad y bondad) y en que es agradable al espíritu (o inteligencia, principalmente), y en esto coincide con los autores medievales. En primer lugar, dice, lo bello, pues, es perfecto en verdad y bondad. Lo bello es, entonces, un trascendental. Antes de aplicarse a las cosas u objetos, lo bello, lo verdadero y lo bueno, aunque distinguibles, coinciden con el ser. Este acercamiento es metafísico. En segundo lugar, agrega que lo bello es lo que agrada al espíritu, es decir, no puede ser bello lo que no agrada. Lo bello en general, puede decirse, lo que comparten todas las cosas bellas y estas mismas, son bellas porque agradan al espíritu, se puede inferir que primero son bellas. Ya se está en el ámbito de la sensibilidad. Ya en el ámbito de las artes, dice. En tercer lugar, el padre PJM dice que hay belleza en los objetos cuando son perfectos (o acabados o completos). A esto se suma, por supuesto, el agrado del espíritu movido por dicha perfección. Así pues, metafísicamente, lo bello es lo perfecto, los objetos bellos, las piezas de arte, lo son porque son perfectos y esto mueve al espíritu a sentir agrado. Aquí tenemos desdoblada, pero en líneas generales, la Estética de PJM en una filosofía de la belleza, una filosofía del arte y una filosofía de la sensibilidad.

\section{Referencias}

Arvisu, A. (2005). Apuntes de Estética. Querétaro: OMNES HOMINES.

Astorgano, A. (2015a). La contextualización artística del P. Pedro José Márquez en el jesuitismo expulso. IHS. Antiguos Jesuitas en Iberoamérica, 3(2), 191-205.

Astorgano, A. (2015b). El clasicismo en la época de PJM (1741-1820). Arqueología, filología, historia, música y teoría arquitectónica. En O. Flores (Coord.). IHS. Antiguos Jesuitas en Iberoamérica, 3(2), 260-265. México, Universidad Nacional Autónoma de México, Instituto de Investigaciones Estéticas / Real Academia de Bellas Artes de San Fernando, 2014, pp. 645. 
Beuchot, M. (1990). Panorama de la historia de la filosofía Novohispana. Discurso como miembro de la Academia Mexicana de la Historia, leído por el dr. Mauricio Beuchot Puente, el 29 de mayo de 1990.

Beuchot, M. (1996). Historia de la filosofía en el México colonial. Barcelona: Herder.

Beuchot, M. (2009). Tratado de hermenéutica analógica. México: UNAM.

Beuchot, M. (2012). Belleza y analogía. Una introducción a la Estética. México: San Pablo.

Blanco, P. (2007). Estética de bolsillo. Madrid: Palabra.

Fernández, J. (1972). PJM en el recuerdo y en la crítica. Márquez, Pedro José (17411820). En F. Justino (Ed.). Sobre lo bello en general y dos monumentos de arquitectura mexicana Tajín y Xochicalco. México: UNAM-Instituto de Investigaciones Filológicas.

Fernández, M. (2011). Literatura artística en el siglo XVIII. En N. Vogeley y M. Ramos (Coords.). Historia de la literatura mexicana 3. Cambios de reglas, mentalidades y recursos retóricos en la Nueva España del siglo XVIII, (525-548). México: UNAMSiglo XXI Editores.

Flores, Ó. (2010). Reseña de "El clasicismo en la época de PJM (1741-1820). Arqueología, filología, historia, música y teoría arquitectónica”. Boletín de Monumentos Históricos, INAH, (20), 223-225. México: Tercera Época.

Ibargüengoitia, A. (1982). Filosofía mexicana en sus hombres y en sus textos. México: Porrúa (colección Sepan cuántos...).

Ibargüengoitia, A. (1989). Suma filosófica mexicana. México: Porrúa (colección Sepan cuántos...).

Larroyo, F. (1989). La filosofía iberoamericana. Historia, formas, temas, polémica, realizaciones. México: Porrúa (colección Sepan cuántos...).

Márquez, P. J. (1801). Sobre lo bello en general. Discurso de Don PJM, presbítero, socio de las Academias de Bellas Artes de Madrid, de Florencia y de Bolonia, a un amigo. Con la licencia en la oficina del Diario. 
114 Juan Granados Valdéz

Navarro, B. (1998). Filosofía y cultura novohispanas. México: UNAM-Instituto de Investigaciones Filosóficas.

Rovira, C. (2014). Ontología de un humanismo salvador. Estudios 110, 12, 105-128. Otoño. 